\title{
DERECHOS SEXUALES Y REPRODUCTIVOS EN ARGENTINA: NUEVAS TENDENCIAS LEGISLATIVAS Y OPINIÓN DE LOS MÉDICOS
}

Sexual and reproductive rights in Argentina: New legislative trends and physician opinions

${ }^{1}$ Universidad Nacional de Córdoba. Córdoba, Argentina.

Correspondência: Maximiliano Nicolás Campana. E-mail: maxicampana@gmail.com.

Recebido em: 21/09/2016. Revisado: 24/02/2017. Aprovado: 07/03/2017. 


\section{RESUMO}

Si trazamos una trayectoria del derecho sanitario argentino en la última década, podríamos ver cómo la nueva legislación otorga cada vez mayor centralidad a la autonomía de la voluntad los pacientes. Los ciudadanos argentinos cada vez tienen mayor poder de decisión en todo lo relativo al cuidado de su propio cuerpo y salud. Esto sin dudas provoca un corrimiento del rol tradicional que ocupaban los médicos, quienes históricamente tomaban las decisiones relativas al cuidado de la salud de la población. El presente artículo se va a enfocar en cómo los derechos sexuales y reproductivos comienzan a ser regulados en el país y luego contrastar la actualidad legislativa con la opinión de médicos que viven en la ciudad de Córdoba (Argentina), con la finalidad de ver de qué modo los profesionales de la salud se adecúan o no a estas nuevas tendencias legislativas.

\section{Palabras claves}

Aborto; Derecho à la Salud; Derechos Sexuales y Reproductivos; Identidad de Género.

\section{ABSTRACT}

If tracing the trajectory of right to health in Argentina over the last decade, we can see how the new legislation grants increasing importance to the voluntary will of patients. Argentine citizens are increasingly able to make decisions regarding everything related to the care of their own bodies and health. This undoubtedly causes a shift in the traditional role of physicians, who historically have made decisions regarding healthcare for the population. This article will focus on how sexual and reproductive rights have started to be regulated in the country, and then compare the current legislation with the opinion of doctors living in the city of Cordoba (Argentina), to see if healthcare professionals are in accordance with these new legislative trends.

\section{Keywords}

Abortion; Right to Health; Sexual and Reproductive Rights; Gender Identity. 


\section{Introducción}

El 9 de mayo del año 2012 la Cámara de Senadores de la República Argentina sancionaba dos leyes que serían importantísimas en materia de reconocimiento de la autonomía de la voluntad de los pacientes sobre su propio cuerpo. Estas serían las leyes $26.742^{1}$ - que regula temas vinculados a la eutanasia pasiva, también denominada de "muerte digna", en el marco de la ley de derecho de los pacientes - y $26.743^{2}$ - denominada como "ley de identidad de género", norma que reconoce el derecho a la identidad autopercibida de género de personas transexuales, sin diagnósticos médicos previos o autorizaciones judiciales -, profundizando una tendencia legislativa que se viene vislumbrando en el país en materia de derecho a la salud: el rol cada vez más relevante de la autonomía de la voluntad de los pacientes sobre sus propios cuerpos.

Junto a las leyes de "muerte digna" e "identidad de género", podemos mencionar otras normas nacionales que se encontrarían en un mismo paradigma, tales como la $25.673^{3}$ - Programa Nacional de Salud Sexual y Procreación Responsable; $25.929^{4}$ - ley de parto respetado/humanizado; 26.529 - ley de derecho de los pacientes; y $26.862^{5}$-ley nacional de fertilización asistida. Todas estas normas además de regular cuestiones de derecho sanitario, reconfiguran diversas relaciones, como son aquellas de "médicos/pacientes"; "consumidores/mercado farmacéutico" y las ideas mismas de "sano/enfermo", colocando a los ciudadanos/ pacientes y a la libre voluntad expresada por ellos en un lugar central dentro de este nuevo paradigma legal.

Si bien el concepto clásico y quizás el más utilizado para definir la salud es el brindado por la Organización Mundial de la Salud (OMS) es decir, entender salud como "un estado de completo bienestar físico, mental y social, y no solamente

${ }^{1}$ ARGENTINA. Ley 26.742, de 24 de mayo de 2012. Ley sobre derechos del paciente, historia clínica y consentimiento informado. Disponible en: <http://www.psi.uba.ar/academica/carrerasdegrado/ psicologia/sitios_catedras/obligatorias/723_etica2/material/normativas/ley_26742_muerte_digna. pdf>. Acceso en: 31 mayo 2017 .

${ }^{2}$ ARGENTINA. Ley 26.743, de 23 de mayo de 2012. Establécese el derecho a la identidad de género de las personas. Disponible en: <http://www.ms.gba.gov.ar/sitios/tocoginecologia/files/2014/01/Ley-26.743IDENTIDAD-DE-GENERO.pdf>. Acceso en: 31 mayo 2017.

${ }^{3}$ ARGENTINA. MINISTERIO DE LA SALUD. Ley 25.673, de 26 de mayo de 2003. Programa Nacional de Salud Sexual y Procreación Responsable. Disponible en: <http://www.msal.gob.ar/saludsexual/ley.php>. Acceso en: 31 mayo 2017.

${ }^{4}$ ARGENTINA. MINISTERIO DE JUSTICIA Y DERECHOS HUMANOS. Ley 25.929, de 17 de septiembre de 2004. Establécese que las obras sociales regidas por leyes nacionales y las entidades de medicina prepaga deberán brindar obligatoriamente determinadas prestaciones relacionadas con el embarazo, el trabajo de parto, el parto y el postparto, incorporándose las mismas al Programa Médico Obligatorio. Derechos de los padres y de la persona recién nacida. Disponible en: <http://servicios.infoleg.gob.ar/infoleglnternet/ anexos/95000-99999/98805/norma.htm>. Acceso en: 31 mayo 2017.

${ }^{5}$ ARGENTINA. MINISTERIO DE JUSTICIA Y DERECHOS HUMANOS. Ley 26.862, de 25 de junio de 2013. Acceso integral a los procedimientos y técnicas médico-asistenciales de reproducción médicamente asistida. Disponible en: <http://servicios.infoleg.gob.ar/infolegInternet/anexos/215000-219999/216700/norma. htm >. Acceso en: 31 mayo 2017. 
la ausencia de afecciones o enfermedades"', en general el sistema sanitario argentino se ha basado en ser principalmente patologizante: la salud se entendía como la ausencia de enfermedad, y la preexistencia de una enfermedad, y el consiguiente reconocimiento estatal de la misma, permitía a ciudadanos y ciudadanas obtener acceso al sistema sanitario del país ${ }^{7}$. La inclusión en el Programa Médico Obligatorio $(\mathrm{PMO})^{8}$ de determinadas patologías era y continúa siendo central para hacer efectivo ese derecho a la salud.

Sin embargo, estas nuevas tendencias legislativas parecen entender a la salud como algo más que la mera ausencia de enfermedades: ya no es necesario ser declarados enfermos por profesionales de la salud para acceder a determinadas prestaciones médicas, porque el bienestar social y psíquico también forman parte de este derecho la salud de todos los individuos. De este modo, la ley $25.673^{9}$ que instaura el Programa Nacional de Salud Sexual y Procreación Responsable, establece que, ante la simple solicitud de cualquier persona interesada se podrá "suministrar los métodos y elementos anticonceptivos que deberán ser de carácter reversible, [...]; Aceptándose además la [...] ligadura de trompas de Falopio y [...] vasectomía, requeridas formalmente como método de planificación familiar y/o anticoncepción" (artículo. 6, inciso b) ya que entre el primer objetivo de esta ley está "alcanzar para la población el nivel más elevado de salud sexual y procreación responsable con el fin de que pueda adoptar decisiones libres de discriminación, coacciones o violencia" (artículo 2, inciso a). Esta ley incluye el acceso a métodos anticonceptivos a menores de edad (artículo 4), y el decreto que lo reglamenta (1.282/2003), en su artículo cuatro establece que

a los efectos de la satisfacción del interés superior del niño, considéreselo al mismo beneficiario, sin excepción ni discriminación alguna, del más alto nivel de salud y dentro de ella de las políticas de prevención y atención en la salud sexual y reproductiva en consonancia con la evolución de sus facultades ${ }^{10}$.

${ }^{6}$ ORGANIZACIÓN MUNDIAL DE LA SALUD. Disponible en: <http://www.who.int/suggestions/faq/es/>. Acceso en: 31 mayo de 2017.

${ }^{7}$ Quizás uno de los pocos casos de acceso al sistema sanitario sin patologización era el caso de embarazos. Las mujeres embarazadas no se consideraban enfermas, pero sin embargo tenían libre acceso al sistema.

${ }^{8}$ El Programa Médico Obligatorio (PMO) es una canasta básica de prestaciones a través de la cual los beneficiarios tienen derecho a recibir prestaciones médico asistenciales. La obra social debe brindar las prestaciones del PMO y otras coberturas obligatorias, sin carencias, preexistencias o exámenes de admisión. Sobre esto, véase SCERVINO: “La evaluación de tecnologías sanitarias aporta valor y eficacia al sistema de salud. Disponible en: <http://www.sssalud.gov.ar/index/index. php?cat $=$ pmo\&opc $=$ pmoprincipal $>$.

${ }^{9}$ ARGENTINA. MINISTERIO DE LA SALUD. Ley 25.673, de 26 de mayo de 2003, cit.

${ }^{10}$ ARGENTINA. Boletín Oficial de la República de Argentina. Disponible en: <https://www.boletinoficial.gob.ar /\#!DetalleNorma/7253193/20030526>. Acceso en: 31 mayo 2017. 
En este caso,

[...] en las consultas se propiciará un clima de confianza y empatía [...] las personas menores de edad tendrán derecho a recibir, a su pedido y de acuerdo a su desarrollo, información clara, completa y oportuna; manteniendo confidencialidad sobre la misma y respetando su privacidad". Por último, establece que los métodos anticonceptivos serán entregados a menores de 14 años siempre que asistan "con sus padres o un adulto responsable ${ }^{11}$.

Por su parte, la ley $26.742^{12}$ pone la voluntad de los pacientes en un plano central. A partir de entonces, "el paciente tiene derecho a aceptar o rechazar determinadas terapias o procedimientos médicos o biológicos, con o sin expresión de causa, como así también a revocar posteriormente su manifestación de la voluntad" incluyendo a los "niños, niñas y adolescentes" quienes "tienen derecho a intervenir en los términos de la ley $26.061^{13}$ a los fines de la toma de decisión sobre terapias o procedimientos médicos o biológicos que involucren su vida o salud" (artículo 1). Asimismo los pacientes en aquellos casos que padezca alguna enfermedad irreversible, incurable, o terminal tendrá el derecho de rechazar procedimientos de hidratación y alimentación que prolonguen ese estadio terminal (artículo 2, inciso g) ${ }^{14}$. De igual modo, la ley $26.743^{15}$, que regula el reconocimiento a la identidad de género, establece en su artículo 11 que todas las personas podrán acceder "a intervenciones quirúrgicas totales y parciales y/o tratamientos integrales hormonales para adecuar su cuerpo, incluida su genitalidad, a su identidad de género autopercibida, sin necesidad de requerir autorización judicial o administrativa". Y para el acceso a este derecho "se requerirá, únicamente, el consentimiento informado de la persona". Sin embargo, cuando se trate de personas menores de dieciocho años, el acceso a estas intervenciones o tratamientos "deberá ser efectuada a través de sus representantes legales y con expresa conformidad del menor" debiendo éste "contar con la

\footnotetext{
${ }^{11}$ ARGENTINA. Boletín Oficial de la República de Argentina, cit.

${ }^{12}$ ARGENTINA. Ley 26.742, de 24 de mayo de 2012, cit.

${ }^{13}$ Por medio de esta ley se le reconoce a niños, niñas y adolescentes el derecho de ser incluidos en la toma de decisiones, permitiéndole intervenir en las cuestiones que los afectan. ARGENTINA. MINISTERIO DE JUSTICIA Y DERECHOS HUMANOS. Ley 26.061, de 21 de octobre de 2005. Disposiciones generales. Objeto. Principios, Derechos y Garantías. Sistema de Protección Integral de los Derechos de las Niñas, Niños y Adolescentes. Organos Administrativos de Protección de Derechos. Financiamiento. Disposiciones complementarias. Disponible en: <http://servicios.infoleg.gob.ar/infolegInternet/anexos/110000-114999/110778/norma. htm>. Acceso en: 31 mayo 2017.

${ }^{14}$ Cuando los pacientes se encuentren incapaces de brindar su consentimiento, la ley manda a aplicar analógicamente el artículo 21 de la ley 24.193 de "transplante de órganos y materiales anatómicos". En este caso, el consentimiento se será dado por las siguientes personas, respetando este orden: a) El cónyuge no divorciado que convivía con el fallecido, o la persona que sin ser su cónyuge convivía con el fallecido en relación de tipo conyugal no menos antigua de tres (3) años, en forma continua e ininterrumpida; b) Cualquiera de los hijos mayores de dieciocho (18) años; c) Cualquiera de los padres; d) Cualquiera de los hermanos mayores de dieciocho (18) años; e) Cualquiera de los nietos mayores de dieciocho (18) años; f) Cualquiera de los abuelos; g) Cualquier pariente consanguíneo hasta el cuarto grado inclusive; h) Cualquier pariente por afinidad hasta el segundo grado inclusive; i) El representante legal, tutor o curador.

${ }^{15}$ ARGENTINA. Ley 26.743, de 23 de mayo de 2012, cit.
} 
asistencia del abogado del niño". En caso de ausencia de consentimiento, la ley prevé la posibilidad del menor de solicitar autorización judicial a fin de suplir dicha falta de consentimiento (artículo 5).

Finalmente, la ley $26.862^{16} \mathrm{o}$ de fertilización asistida en su artículo 7 establece que "tiene derecho a acceder a los procedimientos y técnicas de reproducción médicamente asistida, toda persona mayor de edad que haya explicitado su consentimiento informado". Conforme a esta ley, el derecho existe independientemente de ser infértil o no, bastando la simple manifestación libre de voluntad para acceder al mismo.

Las normas reseñadas marcan una tendencia legislativa clara: la salud ya no puede ser meramente entendida como la ausencia de la enfermedad, el derecho a la salud se ha visto ampliado enormemente en este último tiempo, y existe cierta tendencia a la despatologización y a una visión más integral, más compleja, relacional y social de la salud. Y en este contexto, el bienestar físico, psíquico y social parece depender de la voluntad y autopercepción de cada paciente, quedando los médicos y los demás profesionales de la salud en un plano diferente al que tradicionalmente ocuparon.

Este nuevo paradigma sanitario nos lleva a preguntarnos cuál es la opinión de los médicos ante esta nueva realidad. El rol médico, tradicionalmente paternalista, generaba relaciones de poder y autoridad sobre las decisiones de los pacientes. Sin embargo, la progresiva diversificación y especialización de la medicina, la importante absorción por parte del mercado- empresas y hospitales privados, por ejemplo - de cuestiones sanitarias, el progresivo deterioro de los sistemas públicos de salud y el mayor conocimiento por parte de los ciudadanos vinculados a temas de salud y bienestar, entre otras causas, han convertido a los médicos y auxiliares de la medicina muchas veces en operadores sanitarios anónimos, burócratas y sin una relación más directa y personal con las personas que atienden. Ante estas cuestiones, estimamos de gran valor indagar sobre las opiniones que distintos médicos y médicas tienen en relación a estos nuevos rumbos legislativos en particular, centrándonos en aquellas normas que se vinculan a temas de derechos sexuales y reproductivos, en donde la autonomía de la voluntad sobre el propio cuerpo adquiere un rol central.

\section{Abordaje metodológico}

Con el fin de abordar esta cuestión, se ha realizado un análisis documental y revisión legislativa, teórica y doctrinaria, por un lado, y análisis cuantitativos, por el otro, con el fin de complementar la información. Para la parte documental, se realizó una extensa revisión bibliográfica con el fin de identificar normas nacionales que coloquen a la autonomía de la voluntad en un plano central cuando se trate de cuestiones vinculadas a derechos sexuales y reproductivos. Sin desconocer

\footnotetext{
${ }^{16}$ ARGENTINA. MINISTERIO DE JUSTICIA Y DERECHOS HUMANOS. Ley 26.862, de 25 de junio de 2013, cit.
} 
la posibilidad de existencia de más normas, se identificaron fundamentalmente cinco leyes nacionales: 25.673 (Programa Nacional de Salud Sexual y Procreación Responsable); 25.929 (parto humanizado); 26.742 (muerte digna y derecho de los pacientes); 26.743 (identidad de género); y 26.862 (fertilización asistida). El análisis de estas leyes se hizo utilizando no sólo los propios textos legales, sino también opiniones doctrinarias, jurisprudenciales y demás aportes teóricos de otras disciplinas relevantes.

Para la parte cuantitativa, se diseñó una encuesta cerrada, anónima, de tipo multiple choice o de opciones múltiples. La encuesta se distribuyó a médicos de la ciudad de Córdoba tanto de manera digital, a través de correo electrónico, como en versión impresa. En total, se obtuvo una muestra de 73 encuestados ${ }^{17}$, y para poder contar con una aproximación demográfica de los mismos se realizaron preguntas vinculadas al sexo y la edad, para con ello poder desagregar datos y ver si existían variaciones importantes en sus respuestas. Recolectado los datos cuantitativos, se prosiguió con el análisis de los mismos y la interpretación de los resultados, a fin de detectar si había variaciones importantes entre la opinión de médicos y lo que regula la ley. Finalmente, se procedió a redactar los resultados obtenidos, tal como se presentan a continuación.

\section{II. ¿Qué entendemos por derechos sexuales y reproductivos?}

Antes de comenzar con el análisis de los resultados, es importante presentar lo que se entiende por "derechos sexuales y reproductivos", a los que definimos como derechos fundamentales que contemplan el respeto a la persona humana en aquello vinculado a la realización plena, segura y libre de su vida sexual, su orientación sexual e identidad de género, a la libre opción de la maternidad/paternidad, y la planificación familiar voluntaria y responsable.

A esta definición la elaboramos a partir de los primeros documentos internacionales relevantes sobre esta cuestión, entre los que se puede destacar, cronológicamente:

1) La Conferencia Mundial de Derechos Humanos (Viena, 1993) que estableció que "[...] los derechos humanos de la mujer y de la niña son parte inalienable, integrante e indivisible de los derechos humanos universales"18.

2) La IV Conferencia Mundial de la Mujer de Naciones Unidas (Beijing, 1995) que estableció que:

\footnotetext{
${ }^{17}$ Es importante aclarar que si bien la muestra no es estadísticamente significativa para confiar en los resultados, ya que la misma debió contar con al menos unas 350 encuestas, tomando como base la población total de médicos matriculados en la ciudad de Córdoba, creemos que da fuertes indicios de cuál podría ser la opinión generalizada de estos profesionales de la salud.

${ }^{18}$ ORGANIZACIÓN DE LAS NACIONES UNIDAS. Conferencia Mundial sobre Derechos Humanos, Viena, 14 al 25 de junio de 1993. Disponible en: <https://documents-dds-ny.un.org/doc/UNDOC/GEN/G93/142/36/ PDF/G9314236.pdf?OpenElement>. Acceso en: 31 mayo de 2017.
} 
La salud reproductiva es un estado general de bienestar físico, mental y social [...] en todos los aspectos relacionados con el sistema reproductivo y sus funciones y procesos. En consecuencia, la salud reproductiva entraña la capacidad de disfrutar de una vida sexual satisfactoria y sin riesgos y de procrear, y la libertad para decidir hacerlo o no hacerlo, cuando y con que' frecuencia. [...] Incluye también la salud sexual, cuyo objetivo es el desarrollo de la vida y de las relaciones ${ }^{19}$.

3) El Programa de Acción de la Conferencia Internacional sobre la Población y el Desarrollo, párrafo 7.2, contenido en el Informe de la Conferencia Internacional sobre la Población y el Desarrollo ${ }^{20}$, Cairo, 5 a 13 de septiembre de 1994, que considera al ser humano no sólo como un ser biológico, aislado de su contexto sino como un ser social, relacionado de manera particular a través de su sexualidad; y a los problemas relativos a la sexualidad y la reproducción como un todo integrado que se debe abordar de ese modo tanto para su promoción, como para su prevención y atención, y por último,

4) La Convención sobre la Eliminación de Todas las Formas de Discriminación contra la Mujer, específicamente en su artículo 12.1 establece que "los Estados Partes adoptarán todas las medidas apropiadas para eliminar la discriminación contra la mujer en la esfera de la atención médica [... inclusive los que se refieren a la planificación de la familia ${ }^{21 ”}$. En el artículo 16, inciso e, dispone que

asegurarán en condiciones de igualdad entre hombres y mujeres los mismos derechos a decidir libre y responsablemente el número de sus hijos y el intervalo entre los nacimientos y a tener acceso a la información, la educación y los medios que les permitan ejercer estos derechos ${ }^{22}$.

El elenco de derechos sexuales y reproductivos, es amplío y variable, pero va adquiriendo cada vez mayor importancia tanto a nivel internacional o en cada país. La necesidad de regular este tipo de derechos surgiría debido a los cambios que se han producido, con relación a la posición que comienza a ocupar la mujer en la sociedad, la estructura familiar y el rol de cada uno de sus miembros y las distintas realidades sociales.

\footnotetext{
${ }^{19}$ ORGANIZACIÓN DE LAS NACIONES UNIDAS. Cuarta Conferencia Mundial Sobre la Mujer, Beijing, 4 a 15 de septiembre de 1995. Disponible en: <http://www.un.org/documents/ga/conf177/aconf177-20sp.htm>. Acceso enj: 31 mayo de 2017.

${ }^{20}$ ORGANIZACIÓN DE LAS NACIONES UNIDAS. Informe de la Conferencia Internacional sobre la Población y el Desarrollo, Cairo, 5 a 13 de septiembre de 1994. Disponible en: <https://documents-dds-ny.un.org/doc/ UNDOC/GEN/N95/231/29/PDF/N9523129.pdf?OpenElement>. Acceso enj: 31 mayo de 2017.

${ }^{21}$ ORGANIZACIÓN DE NACIONES UNIDAS. Convención sobre la Eliminación de Todas las Formas de Discriminación contra la Mujer. Disponible en: <http://www.un.org/womenwatch/daw/cedaw/text/ sconvention.htm>. Acceso enj: 31 mayo de 2017.

${ }^{22}$ Id. Ibid.
} 
En este sentido se ha dicho que

\begin{abstract}
A partir de la década del sesenta, se impone el modelo de familia contemporánea o posmoderna que se presenta como un espacio signado por el pluralismo y la autonomía donde parece no cuestionarse que, en principio, son los propios miembros de la familia quienes se encuentran en mejores condiciones para decidir respecto de los asuntos que los afectan ${ }^{23}$.
\end{abstract}

Dentro de este contexto se produce una paradoja: por un lado asistimos a la creciente privatización de la familia, que consiste en el hecho de que la sociedad contemporánea tiende a representar y a legitimar a la familia esencialmente como ámbito de las relaciones expresivo-comunicativas libres de cualquier tipo de responsabilidad pública. Por el otro, se produce el fenómeno de la publicización de la familia, es decir, el creciente interés del Estado en regular los derechos de los individuos y las relaciones en la familia de modo cada vez más detallado, sustancial e interno, en lugar de hacerlo según las modalidades generales, formales y exteriores ${ }^{24}$.

Lo cierto es que el Estado Argentino viene regulando sobre la salud sexual y reproductiva de la población desde un paradigma de derechos humanos, donde, como venimos sosteniendo, la autonomía de la voluntad de los pacientes ocupa un lugar central, algo que se torna aún más evidente en materia de derechos sexuales y reproductivos. Con esto en mente, nos enfocaremos en presentar y analizar tres cuestiones vinculadas a la salud sexual y reproductiva: fertilización asistida, identidad de género e interrupción voluntaria del embarazo (aborto), contrastando la normativa vigente con la opinión de algunos médicos de la ciudad de Córdoba.

\title{
1. Ley 26.862 sobre acceso a procedimientos de reproducción asistida
}

El 26 de Junio de 2013 se publicó en Argentina la ley nacional 26.862 25 , conocida como la "Ley de Fertilización Asistida". El objetivo de la misma es garantizar "el acceso integral e igualitario a los procedimientos y técnicas médico-asistenciales de reproducción médicamente asistida", considerando que incluye los "procedimientos y técnicas realizados con asistencia médica para la consecución de un embarazo, comprendiendo todo tipo de técnicas ofrecidas por la ciencia médica”. En este sentido, la norma establece que toda persona mayor de edad tiene derecho a acceder estos procedimientos siempre que haya explicitado su consentimiento informado, el cual puede ser revocado hasta antes de la implantación del embrión. Una cuestión interesante es que la norma no requiere la condición de infertilidad o

\footnotetext{
${ }^{23}$ FAMÁ, María Victoria; REVSIN, Marisa Herrera y Moira. Un paso más hacia su consolidación... Los derechos sexuales y reproductivos como un derecho humano. Revista La Ley Córdoba, Buenos Aires, p. 800, Sept. 2004. ${ }^{24}$ SESTA, Michele. Lo privado y lo público en los proyectos de ley en materia de familia. In: CARLUCCl, Aída Kemelmajer (Coord.). El derecho de familia y los nuevos paradigmas. Buenos Aires: Rubinzal-Culzoni Editores, 1999. t. 1. ${ }^{25}$ ARGENTINA. MINISTERIO DE JUSTICIA Y DERECHOS HUMANOS. Ley 26.862, de 25 de junio de 2013, cit.
} 
la imposibilidad de concebir un embarazo para poder ser beneficiario. Entre otras cuestiones, la ley crea un registro único de establecimientos médicos, públicos y privados, habilitados para realizar estos procedimientos, estableciendo además que las mismas deberán ser brindadas por los establecimientos públicos de salud, las obras sociales, las entidades de medicina prepaga y, en general, todos los agentes que brinden servicios médico-asistenciales, con independencia de la figura jurídica que posean. Por último la norma aclara que la autoridad de aplicación no podrá introducir requisitos o limitaciones que impliquen la exclusión debido a la orientación sexual o el estado civil de los destinatarios.

La finalidad de esta norma era universalizar el acceso a técnicas de fertilización asistida de parejas que no contaran con los medios suficientes para por acceder a ellas. La universalización trajo consigo dos consecuencias: la primera es que deba ser todo el sistema de salud, tanto público como privado, quien deba hacerse cargo de estas terapias, en general muy costosas, sobrecargando financiera y logísticamente un ya saturado sistema público de salud. La segunda es que cualquier persona pueda acceder a las prestaciones de la ley, sin necesidad de diagnósticos médicos que dictaminen su infertilidad. En los hechos concretos, esto permitiría acceder a estas técnicas tanto a mujeres solteras, como a parejas de mujeres del mismo sexo que quisieran quedar embarazadas, y aun no siendo infértiles, no deseen concebir a través de relaciones sexuales heterosexuales. Lo cierto es que a dos años de aprobada la ley, parece que la misma no tuvo el impacto esperado en muchas parejas que quisieron acceder al mismo, ya que según los resultados de una encuesta, "el $42 \%$ de los encuestados no logró todavía ninguna cobertura para los tratamientos y el 39\% consiguió, apenas, acceso a una cobertura parcial". Además, "el $24 \%$ aún no pudo realizar ningún tratamiento de reproducción" y "el $67 \%$ de la muestra no realizó el reclamo ante la Superintendencia de Servicios de Salud y que nada más que el 9\% inició acciones legales -como amparos- reclamando por la cobertura" ${ }^{26}$.

Ahora bien, teniendo en cuenta la norma, y los desafíos que la misma genera, una de las preguntas realizadas a los médicos fue “¿stá usted de acuerdo con que los hospitales públicos, las obras sociales y las empresas de medicina privada deban hacerse cargo de tratamientos de fertilización asistida en todos los casos, incluso cuando no hay un diagnóstico de infertilidad?". Ante esta pregunta, las respuestas obtenidas fueron las siguientes: el 28,77\% acordó totalmente; hubo un acuerdo parcial el 50,69\% y, por último, el 20,5\% mostró su desacuerdo. Es decir que menos del $29 \%$ de los médicos acuerdan con la cobertura igualitaria que brinda la ley 26.862, y en general los médicos muestran importantes reticencias al momento de reconocer este derecho. De aquellos que fueron encuestados

${ }^{26}$ A DOS AÑOS de la ley de fertilización, lo que todavía falta. Infobase, 06 jun. 2015. Disponible en: <http://www. infobae.com/2015/06/06/1733294-a-dos-anos-la-ley-fertilizacion-lo-que-todavia-falta>. Acceso en: 20 set. 2016. 
personalmente, surgía que la falta total o parcial de acuerdo se debía a considerar que el sistema de salud debía encargarse de temas prioritarios, no siendo la fertilización uno de ellos o que no consideraban que mujeres fértiles debieran acceder a estos tratamientos. Según estos encuestados, debían ser los profesionales de la salud quienes determinen sobre la necesidad de acceder o no a este tipo de procedimientos.

\title{
2. Ley $\mathbf{2 6 . 7 4 3}$ sobre identidad de género
}

La ley de identidad de género $26.743^{27}$ (en adelante, LIG) se trata de un hito legislativo importante en materia de reconocimiento de derechos en el país, pero además, es una normativa novedosa, sin precedentes y que genera una nueva gramática para entender conceptos de identidad, sexualidad, género, salud y autodeterminación sobre el propio cuerpo en el país. La LIG, aprobada con gran consenso en el Congreso de la Nación tiene características únicas que trastoca los modelos jurídicos existentes y nos obliga a repensarnos como ciudadanos.

Para entender el concepto de identidad de género, recurrimos a la definición propuesta por los Principios de Yogyakarta, es decir, los principios sobre la aplicación de la legislación internacional de derechos humanos en relación con la orientación sexual y la identidad de género. En el preámbulo de estos principios se define a la identidad de género como:

\begin{abstract}
la vivencia interna e individual del género, tal como cada persona la siente profundamente, la cual podría corresponder o no con el sexo asignado al momento del nacimiento, incluyendo la vivencia personal del cuerpo (que podría involucrar la modificación de la apariencia o la función corporal a través de medios médicos, quirúrgicos o de otra índole, siempre que la misma sea libremente escogida) y otras expresiones de género, incluyendo la vestimenta, el modo de hablar y los modales (Preámbulo, Quinto párrafo) ${ }^{28}$.
\end{abstract}

Esta definición es la adoptada también por la ley 26.743, en su artículo 2, y si bien en abstracto es suficientemente abarcativa para incluirnos a todos/as en la misma, en el sentido que todos/as tenemos nuestra propia identidad de género, lo cierto, y como señala Mauro Cabral ${ }^{29}$ la definición está pensando concretamente en reconocerle este derecho a un grupo claramente identificado: el de las personas trans e intersex.

\footnotetext{
${ }^{27}$ ARGENTINA. Ley 26.743, de 23 de mayo de 2012, cit.

${ }^{28}$ Principios de Yogyakarta. Disponible en: <http://www.yogyakartaprinciples.org/principles-sp/>. Acceso en: 31 mayo 2017.

${ }^{29}$ CABRAL, Mauro. Algo ha pasado. In: MORÁN FAÚNDES, José Manuel, SGRÓ RUATA, María Candelaria; VAGGIONE, Juan Marco (Eds.). Sexualidades, desigualdades y derechos: reflexiones en torno a los derechos sexuales y reproductivos. Córdoba: Ciencia, Derecho y Sociedad, 2012.
} 
A partir de la LIG existe legislativamente un derecho a la identidad de género autopercibida ${ }^{30}$, el mismo derecho es reconocido por el Estado, y consta de una parte vinculada al reconocimiento registral y otra, de carácter sanitario, sobre el acceso a terapias hormonales e intervenciones quirúrgicas de reasignación sexual.

A grandes rasgos, el texto legal contempla el derecho de todas las personas al reconocimiento de su identidad de género, al libre desarrollo de su persona conforme a su identidad de género y a ser tratada de acuerdo con su identidad de género y, en particular, a ser identificada de ese modo en los instrumentos que acreditan su identidad respecto de el/los nombre/s de pila, imagen y sexo con los que allí es registrada (artículo 1).

Además, se faculta a todas las personas que así lo deseen a rectificar sus datos registrales cuando ellos no coincidan con su identidad de género autopercibida por medio de un trámite sencillo y rápido (tanto para mayores como para menores de edad, según los artículos 6, 7, 8, 9 y 10), como así también a

acceder a intervenciones quirúrgicas totales y parciales y/o tratamientos integrales hormonales para adecuar su cuerpo, incluida su genitalidad, a su identidad de género autopercibida, sin necesidad de requerir autorización judicial o administrativa, las que deberán ser cubiertas por los sistemas públicos de salud ${ }^{31}$.

La LIG se presenta a nivel mundial como una solución legislativa novedosa ante la problemática que atravesó históricamente el colectivo trans, siendo bienvenida por el movimiento argentino por la diversidad sexual, y elogiada incluso por la Organización de las Naciones Unidas $(\mathrm{ONU})^{32}$. En términos generales, se trata de una normativa que trastoca enormemente el sistema jurídico, en aspectos vinculados a la autonomía de la voluntad y de decisión sobre el propio cuerpo, al acceso al sistema de

\footnotetext{
${ }^{30} \mathrm{El}$ criterio legislativo anterior era claramente contrario a este reconocimiento: las leyes 17.132 (que regula el ejercicio de la medicina), 18.248 (que regula el nombre de las personas) y el Código Penal (en cuanto regulaba las lesiones gravísimas) imposibilitaban tanto el reconocimiento de un nombre distinto al asignado al nacer, como el acceso al sistema sanitario para readecuar el cuerpo de las personas trans al de su identidad autopercibida. La única salida legal era la de hacer una presentación judicial, someterse a peritajes psicológicos, socioambientales, psiquiátricos, demostrar esterilidad y relatar una vida de sufrimiento para así convencer al juez sobre la necesidad de obtener una autorización de cambio de sexo. Además de estas trabas legales para obtener el reconocimiento de la identidad de género, no debemos olvidar que las personas tras eran - y en algunos casos lo siguen siendo - criminalizadas a través de códigos de faltas o edictos policiales. ${ }^{31}$ Artículo 11. ARGENTINA. Ley 26.743, de 23 de mayo de 2012, cit.

${ }^{32}$ Así lo publicó el periódico Página 12: “Naciones Unidas felicitó hoy al Estado argentino por la vigencia de la ley que garantiza el respeto a la identidad de género, una normativa pionera en la región y que procura 'garantizar la igualdad, el respeto y la dignidad de las personas trans'”. La felicitación fue transmitida por la Oficina Regional para América del Sur del Alto Comisionado de Naciones Unidas para los Derechos Humanos (Acnudh)."Argentina ha dado un paso importante para garantizar la igualdad, el respeto y la dignidad de las personas trans", señaló Amerigo Incalcaterra, representante regional para América del Sur de Acnudh. La ley, amén de ser pionera en la región, "incorpora estándares internacionales de derechos humanos en términos de accesibilidad, confidencialidad y universalidad", destacó Incalcaterra. ELOGIO a la ley de identidad de género. Página, 12, 26 mayo 2012. Disponible en: <http://www.pagina12.com.ar/ diario/sociedad/3-194931-2012-05-26.html>. Acceso en: 20 set. 2015.
} 
salud, en aspectos de materia de privacidad e intimidad de las personas y en reconocimiento de la diversidad sexual y las expresiones de género. Además, con ella, se incluye la posibilidad del reconocimiento y respeto a la identidad de género de los/as menores de edad. En definitiva, se trata de una ley que es, al menos, altamente controversial.

Como puede ser observado, la cuestión del derecho a la salud y el acceso al sistema sanitario está fuertemente presente en esta normativa. El reconocimiento de la identidad de género autopercibida implica también la obligación, por parte del sistema integral de salud, de atender los reclamos de aquellas personas que deseen modificar su cuerpo y/o genitalidad, a fin de garantizar el acceso al derecho. Además, significa sacar la transexualidad como una patología: no es necesario ser diagnosticado con "disforia de género" por parte de profesionales médicos ni ser acreditada la esterilidad para que este derecho sea reconocido. En el resto del mundo, en materia de reconocimiento de derechos a personas trans el rol de los médicos es central: son quienes "certifican" que poseen algún transtorno psíquico (disforia de género, en términos del DSM IV ${ }^{33}$ ) o esterilidad, requisitos necesarios para este reconocimiento. En este nuevo contexto legal, parecía importante indagar la posición de los profesionales de la salud en materia de acceso a terapias hormonales o cirugías de reasignación sexual sin intervención médica.

Ante la pregunta “¿Está usted de acuerdo con la posibilidad de que una persona pueda acceder a tratamientos hormonales o cirugías de cambio de sexo sin necesidad de un diagnóstico psiquiátrico previo?", el 67,61\% de los encuestados respondieron que no están de acuerdo, el 12,68\% que están de acuerdo sólo en algunos casos, y el 19,72\% están acuerdan esta posibilidad. Estos datos nos permiten inferir que solamente dos de cada diez médicos reconocen que la identidad de género autopercibida basta para poder acceder al sistema sanitario para una readecuación sexual. En este sentido, en general, la idea patologizante que existe sobre las personas trans sigue estando fuertemente enraizado en el discurso e imaginario médico, tal como queda revelado en las respuestas a esta pregunta ${ }^{34}$.

\section{La cuestión del aborto no punible en Argentina}

En materia de salud reproductiva, planificación familiar, y con fines analíticos, preferimos corrernos un poco de lo establecido por la ley $25.673^{35}$, que crea

\footnotetext{
${ }^{33}$ IV Manual diagnóstico y estadístico de los trastornos mentales (en inglés Diagnostic and Statistical Manual of Mental Disorders, DSM) de la Asociación Estadounidense de Psiquiatría (en inglés American Psychiatric Association - APA) contiene una clasificación de los trastornos mentales y proporciona descripciones claras de las categorías diagnósticas, con el fin de que los clínicos y los investigadores de las ciencias de la salud puedan diagnosticar, estudiar e intercambiar información y tratar los distintos trastornos mentales.

${ }^{34}$ Contrariamente a esto, en Campana se muestra cómo la cuestión "patologizante" estuvo casi completamente ausente durante los debates parlamentarios de la LIG. CAMPANA, Maximiliano. Ley de identidad de Género: Su debate legislativo. Actas del XIV Congreso Nacional y IV Latinoamericano de Sociología Jurídica. "Conflictos sociales y confrontaciones de Derechos en América Latina.” , 2013. Versión digital en CD-Rom.

${ }^{35}$ ARGENTINA. MINISTERIO DE LA SALUD. Ley 25.673, de 26 de mayo de 2003, cit.
} 
Programa Nacional de Salud Sexual y Procreación Responsable, para consultar sobre cuestiones vinculadas a interrupciones voluntarias de embarazos en aquellos casos de no punibilidad. El motivo de este corrimiento se debe a que la figura del aborto, vinculado a la salud reproductiva, es la más controversial, y donde la autonomía de la voluntad de la embarazada también cumple un rol fundamental al momento de interrumpir un embarazo.

Es importante recordar que en Argentina la práctica del aborto es considerada un delito, salvo en aquellos casos que el mismo Código Penal (CP) contempla causales de no punibilidad. En este sentido el artículo 86 del Código Penal establece que:

El aborto practicado por un médico diplomado con el consentimiento de la mujer encinta, no es punible: $1^{\circ}$ ) Si se ha hecho con el fin de evitar un peligro para la vida o la salud de la madre y si este peligro no puede ser evitado por otros medios; $2^{\circ}$ ) Si el embarazo proviene de una violación o de un atentado al pudor cometido sobre una mujer idiota o demente. En este caso, el consentimiento de su representante legal deberá ser requerido para el aborto.

De la mera lectura se considera que este artículo establece dos casos en que la interrupción voluntaria de un embarazo no será perseguido penalmente: el del "aborto terapéutico" (inciso $1^{\circ}$ ) y el del "aborto eugenésico" (inciso $2^{\circ}$ ). Pero notemos que hay dos condiciones que deben cumplirse en ambos casos: el aborto debe ser practicado por un médico diplomado y con el consentimiento de la mujer encinta.

Este artículo, a pesar de haber sido incorporado en nuestro ordenamiento legal hace casi $100 a^{0} o^{36}{ }^{36}$, continúa siendo objeto de controversias legales e interpretativas - posiciones que, sin dudas, se encuentran fuertemente influidas por ideas religiosas, morales, éticas, políticas y posturas ideológicas.

En los hechos, los profesionales de la salud se han estado negando a realizar prácticas abortivas, aun cuando estas no sean punibles, sin una autorización

\footnotetext{
${ }^{36}$ Es importante recordar aquí que en 1886 se sanciona el primer Código Penal de Argentina que penalizaba el aborto en todos los casos. Años después, en 1903, el código es reformado por primera vez y establece la no punibilidad en aquellos casos de tentativa de aborto. Los casos de no punibilidad que actualmente se encuentran vigentes surgen de la segunda reforma del código, sancionada en el año 1921. La Comisión de Códigos del Senado argentino que aprobó el nuevo código estaba integrada por los legisladores Joaquín V. González, Enrique del Valle Iberlucea y Pedro A. Garro. Esta comisión explicó que, en lo concerniente al aborto, se basó en el texto del anteproyecto penal suizo de 1916. También entendió que la no punibilidad del aborto terapéutico no necesitaba explicación; mientras que, para los casos de atentado contra el pudor sobre mujeres con discapacidad mental, se justificó con la siguiente cita del abogado y político español Luis Jiménez de Asúa referida al anteproyecto suizo: "Es la primera vez que una legislación va a atreverse a legitimar el aborto con un fin eugenésico, para evitar que de una mujer idiota o enajenada, o de un incesto, nazca un ser anormal o degenerado". Si bien el texto helvético hacía mención al caso del incesto, éste quedó excluido del código argentino. A pesar de varias modificaciones sufridas por este artículo, con la restauración de la democracia e sanciona la Ley 23.077, que retrotrae nuevamente la situación a lo sancionado en 1921. Véase BERGALLO, Paola. Argentina: Achieving fariness despite "Rutinization"? In: YAMIN, Alicia; GLOPPEN, Siri. Litigating Health Rights. Boston: Harvard University Press, 2011.
} 
judicial. Esa situación fáctica no sólo ha dificultado el acceso a interrupciones voluntarias de embarazos no penalizados, sino que le ha implicado al Estado argentino incluso condenas internacionales: Así por ejemplo, a través de la Comunicación $\mathrm{N}^{\circ}$ $1608 / 2007^{37}$, el Estado recibió una condena internacional del Comité de Derechos Humanos de la ONU por no haber garantizado el acceso a un aborto legal a una adolescente con discapacidad mental, embarazada en una violación, que solicitó la práctica en un hospital público bonaerense. El organismo consideró que la obstrucción del aborto permitido por el CP constituyó una violación de los derechos humanos de la joven y ordenó al país a proporcionarle "medidas de reparación que incluyan una indemnización adecuada" y a "tomar medidas para evitar que se cometan violaciones similares en el futuro". El Comité le dio al país 180 días para cumplir con el dictamen.

Dentro de este contexto, la Corte Suprema de Justicia de la Nación (CSJN) decidió pronunciarse sobre otro caso de aborto no punible, de la provincia de Chubut, con el fin de fijar criterios claros de acción ante estas situaciones: lo hizo en el caso F. A. L. s/medida autosatisfactiva. En este caso, la CSJN consideró entre otras cosas que el aborto está permitido y no resulta punible cuando el embarazo es consecuencia de una violación y esto vale para toda mujer. Es un derecho que se debe interpretar en forma amplia y sin distinciones discriminatorias que lo limiten a quienes padecen una discapacidad mental. Interrumpir la gestación producto del abuso sexual tampoco requiere ninguna clase de autorización judicial ni trámite burocrático. Asimismo, advierte que cualquier otra persona que intente obstaculizar el acceso a un caso de aborto no punible pone las mujeres en una situación de violencia institucional. De ese modo, el máximo tribunal exhortó a funcionarios de la justicia y médicos a dejar de judicializar estos casos, por considerarla una práctica innecesaria, ilegal y cuestionable porque no sólo obliga a la víctima del delito a exponer públicamente su vida privada sino que también la demora que apareja pone en riesgo tanto el derecho a la salud de la solicitante como su derecho al acceso a la interrupción del embarazo en condiciones seguras. Por último, la sentencia conmina a las autoridades nacionales, provinciales y de la ciudad de Buenos Aires a aplicar normas de alto nivel y protocolos hospitalarios para atender los abortos no punibles y para la asistencia integral de toda víctima de violencia sexual.

El caso F. A. L., que pretendía fijar estándares interpretativos claros, concretos y uniformes para todo el país en materia de abortos no punible generó importantes resistencias en diversos tribunales nacionales, mostrando que el caso, lejos de solucionar el problema, lo profundizó. En el caso de la Provincia de Córdoba, por ejemplo, el Ministerio de Salud de la Provincia, como consecuencia

\footnotetext{
${ }^{37}$ Se puede acceder aldictamen completo en ORGANIZACIÓN DE LAS NACIONES UNIDAS. Comité de Derechos Humanos. Pacto Internacional de Derechos Civiles y Políticos. 14 de marzo a 1 de abril de 2011. Disponible en: <http://www.despenalizacion.org.ar/pdf/Jurisprudencia/Argentina/DictamenLMR-CIDH.pdf>. Acceso en: 14 set. 2016.
} 
del caso F. A. L., dictó una normativa - la Guía de Procedimiento para la Atención de Pacientes que soliciten Prácticas de Aborto no Punibles - con el fin de hacer frente a las eventuales solicitudes de interrupciones voluntarias de embarazos dentro de los nuevos estándares fijados por la CSJN. Con esta norma en vigencia, la Asociación Civil "pro-vida" ${ }^{38}$ Portal de Belén se presentó ante los tribunales provinciales, y en representación de todos los niños por nacer solicitó, con éxito, impedir su aplicación a todos los hospitales provinciales (públicos y privados). Situaciones similares se llevaron a cabo en otros lugares. En Buenos Aires, un abogado perteneciente a la Corporación de Abogados Católicos interpuso una acción judicial en los tribunales de la Capital para impedir la realización de un aborto no punible en un hospital público. Mientras tanto, en la provincia de la Santa Fe algunos grupos "pro-vida" junto al Partido Demócrata Cristiano conseguían que se prohibieran las prácticas de aborto no punibles en toda la provincia.

La discusión jurídica del aborto está íntimamente relacionada con el derecho sanitario y el acceso a la salud. Según datos aportados por Sedgh et al..$^{39}$ uno de cada cinco embarazos termina en aborto en el mundo, pero en los países con legislaciones más restrictivas la mayoría de esos procedimientos son inseguros. En un estudio realizado en 160 países, con distintos niveles de desarrollo, se encontró que a mayor apoyo legal hacia el aborto, menor incidencia del aborto inseguro en las tasas de mortalidad materna. Además, según las últimas estimaciones realizadas por la OMS indican que en el mundo se practican aproximadamente 42 millones de abortos inducidos por año (2011). En América Latina y el Caribe, la tasa anual estimada de aborto inseguro en 2008 fue de 31 abortos por cada 1.000 mujeres, mientras que la tasa de procedimientos seguros fue menos de 2 por 1.000. Durante este mismo año, en la región más de 1.100 mujeres murieron a causa de complicaciones derivadas de abortos inseguros, lo que representa el $12 \%$ de la mortalidad materna ${ }^{40}$. En este contexto, no es un dato menor que, según el Observatorio de Salud Sexual y Reproductivo ${ }^{41}$, Argentina, junto a Jamaica y Trinidad y Tobago son los tres países de la región que tienen al aborto como la primera causa de mortalidad materna, donde las complicaciones relacionadas con el aborto superan el $30 \%$ del total de muertes maternas.

\footnotetext{
${ }^{38}$ Por grupos "pro-vida" entendemos a aquellos grupos, organizaciones, asociaciones o movimientos cuyo principal objetivo es llevar a cabo prácticas y políticas tendientes a evitar o subvertir el avance de derechos sexuales, reproductivos y no reproductivos, como así también a promover y defender determinadas nociones de matrimonio (heterosexual, conyugal, monogámico y reproductiva), de familia y de vida (desde la concepción hasta la muerte natural).

${ }^{39}$ SEGHD, Gilda; SINGH, Susheela; SHAH, Iqbal H.; AHMAN, Elisabeth; HENSHAW, Stanley K.; BANKOLE, Akinrinola. Induced abortion: incidence and trends worldwide from 1995 to 2008. The Lancet, London; New York; Beijing, 19 ene. 2012. London; New York; Beijing. <http://www.guttmacher.org/pubs/journals/ Sedgh-Lancet-2012-01.pdf>.

40UNSAFE Abortion: global and regional estimates of the incidence of unsafe abortion and associated mortality in 2008. $6^{\text {th }}$ ed. Ginebra: Organización Mundial de la Salud, 2011.

${ }^{41}$ LA SITUACIÓN de la mortalidad materna en la Argentina. Hoja informativa, Buenos Aires: Observatorio de Salud Sexual y Reproductiva, n. 1, abr. 2010. Disponible en: <http://www.8300.com.ar/wp-content/ uploads/2010/05/hoja_1.pdf>. Acceso en: 21 set. 2016.
} 
En este sentido, son particularmente relevantes las observaciones realizadas por Ramón Michel:

\begin{abstract}
Estas tasas de mortalidad y morbilidad materna también tienen un impacto en la salud pública. Poniendo el foco en los costos, sabemos que el aborto inseguro genera costos -innecesarios- a la sociedad y a los sistemas de salud [...]. Primero, los costos derivados de la atención de las complicaciones médicas derivadas del aborto inseguro constituyen una carga financiera pesada para los sistemas de salud pública en los países en desarrollo [...]. Así, por ejemplo, en la Argentina entre el 20\% y 40\% de las camas de los servicios de ginecología y obstetricia están ocupadas por mujeres que ingresaron al hospital por abortos inseguros [...]. Segundo, las complicaciones post aborto son una de las principales causas de morbilidad materna $[\ldots]^{42}$.
\end{abstract}

Como se puede apreciar hasta ahora, la cuestión del aborto, incluso en los casos de no punibilidad, genera importantes discusiones y resistencias. Es por ello que nos interesaba saber cuál era la opinión de distintos profesionales de la salud en materia de abortos no punibles.

Para ello, se realizaron dos preguntas de opinión. La primera se vinculaba al aborto terapéutico, caso de violación, y la segunda vinculada al aborto eugenésico, caso de riesgo a la salud.

Puntualmente se preguntó si el/la profesional estaba de acuerdo con la interupción de un embarazo no deseado en caso de violación, a lo que el 53,85\% de todos los consultados se manifestaron a favor de la misma. El 28,21\% consideró que sólo debía proceder ante determinados casos, y el 17,95\% se mostró en desacuerdo total con la práctica del aborto aún en este caso.

En relación a los casos de aborto eugenésico, los niveles de acuerdo se modifican sustancialmente (concretamente se preguntó ¿está usted de acuerdo con la interrupción de un embarazo en caso de que la salud de la mujer corra riesgo?). Ante esta pregunta, hay un menor nivel de acuerdo en relación a la práctica en todos los casos $(51,28 \%)$, pero la diferencia se nota en el menor nivel de desacuerdo (sólo el 5,3\%) y un mayor nivel de acuerdo para determinados casos. La diferencia podría deberse al hecho de que los médicos sienten un mayor compromiso en torno al cuidado de la salud de la madre, cuando se producen complicaciones propias del embarazo, que cuando éste es producto de una violación, y dónde, no habiendo complicaciones, la decisión de interrumpir un embarazo depende solamente de la voluntad de la embarazada, y no del médico.

\footnotetext{
${ }^{42}$ RAMON MICHEL, Agustina. Aborto ¿según quién? In: MORÁN FAÚNDES, José Manuel, SGRÓ RUATA, María Candelaria; VAGGIONE, Juan Marco (Eds.). Sexualidades, desigualdades y derechos: reflexiones en torno a los derechos sexuales y reproductivos. Córdoba: Ciencia, Derecho y Sociedad; Ferreyra Editores, 2012.
} 
Por otra parte, además de los datos generales, nos interesaba conocer cuál era la opinión de las mujeres en relación a estas preguntas, debido a que la figura del aborto se trata de un tipo penal que criminaliza determinados cuerpos: los cuerpos femeninos. Para ello, desagregamos la información recolectada, para ver si encontrábamos diferencias. Con relación a la primera pregunta, las diferencias no son sustanciales a la general, aunque la distancia entre el acuerdo parcial y el desacuerdo se acorta: El 52,63\% de las consultadas mostraron un acuerdo total, el 26,32\% un acuerdo parcial y el $21,05 \%$ mostraron un desacuerdo. Sin embargo, ante la cuestión de peligro a la salud, los porcentajes varían fuertemente, y si bien los niveles de desacuerdo se mantienen similares $(5,26 \%)$, las médicas consideran en mayor proporción que sólo en determinados casos se deben realizar abortos en casos de riesgo a la salud (52,63\%), mientras el $42,11 \%$ lo entiende que se debe realizar en todos los casos.

En este sentido, es importante destacar que en relación a este tema, los profesionales de la salud se han manifestado en contra del aborto en mayor proporción que la población argentina. Según el estudio llevado a cabo por el grupo Catholic for choice en el año 2011, más del 80 por ciento de la población se manifestó a favor del acceso de las mujeres al aborto en casos de peligro en la salud de la madre o violación ${ }^{43}$.

Por último, y en relación al aborto, se formuló la siguiente pregunta: ¿usted está de acuerdo con criminalizar a una mujer que se practica un aborto clandestino? La pregunta está vinculada con el hecho de que casi no existen condenas por prácticas de abortos clandestinos en el país, y en general, existe altos niveles de acuerdo con que no se deberían penalizar a las mujeres.

En este sentido, Ramón Michel menciona que "cuando se compara el número de abortos realizados con el número de denuncias, juicios y condenas por aborto, resulta evidente que la herramienta penal es ineficaz para desalentar a las mujeres en su decisión de interrumpir un embarazo" ${ }^{44}$. Según cifras oficiales del 2011 , se estima que en el país se efectúan entre 450.000 y 500.000 abortos por año, casi un 40 por ciento de los embarazos totales. Esta problemática no es menor, por lo que pareció oportuno ver la opinión de los profesionales de la salud ante este tema.

Los resultados son, sin dudas, interesantes. Uno de cada cuatro médicos $(25,64 \%)$ está de acuerdo con penalizar a la mujer que se practique un aborto. Un tercio de los médicos $(33,33 \%)$, considera que sólo en algunos casos debe proceder la pena, y el 41,03\% está en desacuerdo con lo planteado. Cuando la pregunta es

\footnotetext{
${ }^{43} \mathrm{El}$ informe completo puede consultarse online en CATHOLIC for choice. Disponible en: <http://www. catholicsforchoice.org/news/pr/2011/documents/EncuestaenArgentina-ESPANOL.pdf>. Acceso en: 21 set. 2016. Este informe es coincidente con el llevado a cabo por Mónica Petracci en 2007, titulado Opinión pública sobre interrupción voluntaria del embarazo y despenalización del aborto en la Argentina y en América Latina. Informe disponible en PETRACCI, Mónica. Opinión pública sobre interrupción voluntaria del embarazo y despenalización del aborto en la Argentina y en América Latina. 2007. Disponible en: <http:// www.despenalizacion.org.ar/pdf/hojas_informativas/01_petracci.pdf>. Acceso en: 24 fev. 2017.

${ }^{44}$ RAMON MICHEL, Agustina. op. cit.
} 
respondida por mujeres, hay algunas variaciones y menor nivel de acuerdo $(21,05 \%$ a favor, $36,84 \%$ en contra y $42,11 \%$ para determinados casos).

En relación a esta última pregunta, es llamativo el poco acuerdo que hay en relación a la no criminalización de la mujer. La posición mayoritaria nos muestra que los profesionales médicos consideran al aborto como una conducta ilegal y que debe ser castigada. El dato no es menor cuando se piensa en el elevadísimo número de abortos clandestinos estimados que se producen en el país. Así mismo cabe mencionar que estos datos pueden ser contrastados con los arrojados por el informe llevado a cabo por Mónica Petracci ${ }^{45}$, que muestran que el $62 \%$ de la población está en contra de que la mujer sea penada tras practicarse un aborto.

\section{Conclusión}

Tal como mencionamos al comienzo de este artículo, la normativa en materia sanitaria en Argentina tiene una dirección clara: reconocer la autonomía de la voluntad de los ciudadanos, haciendo que ellos sean quienes tomen las decisiones sobre su salud y su propio cuerpo. Estas nuevas normas se enrolarían en un paradigma más amplio, de derechos humanos, donde la salud no se entiende ya como la ausencia de dolencias o enfermedades, sino como el completo bienestar psicofísico.

Pero estas nuevas leyes son también el resultado de una nueva concepción del sistema sanitario. A diferencia de lo que sucedía varias décadas atrás, tanto el rol del médico como de los demás profesionales e instituciones de la salud han comenzado a ser cuestionados y su saber y obrar profesional ya no son considerados indiscutibles. Por el contrario, los pacientes cada vez más asumen el rol de consumidores y las prestaciones médicas como relaciones de consumo. De hecho, según Catalina Smulovitz ${ }^{46}$, los juicios por mala praxis profesional en hospitales dependientes del Gobierno de la Ciudad de Buenos Aires, entre 1982 y 1993 crecieron el $1.000 \%$, y que entre 1995 y 1996 las acciones penales por mala práctica médica aumentaron en un $80 \%$.

Ante esta nueva realidad sociojurídica, se creyó oportuno indagar sobre la opinión de médicos, quienes sin dudas vienen perdiendo terreno en varios aspectos, como son la autoridad profesional y el respaldo social con los que tradicionalmente gozaban. Y a partir de este trabajo exploratorio, los resultados obtenidos son interesantes: claramente, los médicos y médicas encuestados han mostrado un fuerte apego al rol tradicional y parternalista, como así también altos niveles de desacuerdo en relación a conceder a los pacientes elevados márgenes de autonomía para decidir sobre su propio cuerpo y su salud. Estas posturas

\footnotetext{
${ }^{45}$ PETRACCI, Mónica. op. cit.

${ }^{46}$ SMULOVITZ, Catalina. La política por otros medios. Judicialización y movilización legal en la Argentina. Desarrollo Económico, Buenos Aires, v. 48, n. 190/191, p. 287-305. 2008.
} 
mayoritarias chocan fuertemente con las tendencias sociales y particulamente legislativas que analizamos (las que se alejan de una mirada "médicocentrista" y otorgándole un rol fundamental a la autonomía del paciente) generando la impresión que el cambio de paradigma legal no se ve reflejado en el paradigma profesional dominante.

Por último, no se quería dejar de insistir en que si bien se trató de una muestra menor, y no lo suficientemente representativa para realizar afirmaciones categóricas de tipo general sobre la opinión de médicos en torno a estos temas, se espera haber podido contribuir con estos datos, de algún modo, a realizar nuevas preguntas de investigación y encarar nuevas pesquisas sobre la temática.

\section{Referencias}

BERGALLO, Paola. Argentina: Achieving fariness despite "Rutinization"? In: YAMIN, Alicia; GLOPPEN, Siri. Litigating Health Rights. Boston: Harvard University Press, 2011.

CABRAL, Mauro. Algo ha pasado. In: MORÁN FAÚNDES, José Manuel, SGRÓ RUATA, María Candelaria; VAGGIONE, Juan Marco (Eds.). Sexualidades, desigualdades y derechos: reflexiones en torno a los derechos sexuales y reproductivos. Córdoba: Ciencia, Derecho y Sociedad, 2012.

CAMPANA, Maximiliano. Ley de identidad de Género: Su debate legislativo. In Actas del XIV Congreso Nacional y IV Latinoamericano de Sociología Jurídica. "Conflictos sociales y confrontaciones de Derechos en América Latina.” Versión digital en CD-Rom, (2013).

ELOGIO a la ley de identidad de género. Página, 12, 26 mayo 2012. Disponible en: $<$ http://www.pagina12.com.ar/diario/sociedad/3-194931-2012-05-26.html>. Acceso en: 20 set. 2015 .

FAMÁ, María Victoria; REVSIN, Marisa Herrera y Moira. Un paso más hacia su consolidación... Los derechos sexuales y reproductivos como un derecho humano. Revista La Ley Córdoba, Buenos Aires, p. 800-815, Sept. 2004.

LA SITUACIÓN de la mortalidad materna en la Argentina. Hoja informativa, Buenos Aires: Observatorio de Salud Sexual y Reproductiva, n. 1, abr. 2010. Disponible en: <http:// www.8300.com.ar/wp-content/uploads/2010/05/hoja_1.pdf>. Acceso en: 21 set. 2016.

PETRACCI, Mónica. Opinión pública sobre interrupción voluntaria del embarazo y despenalización del aborto en la Argentina y en América Latina. 2007. Disponible en: $<$ http://www.despenalizacion.org.ar/pdf/hojas_informativas/01_petracci.pdf $>$. Acceso en: 24 fev. 2017.

RAMON MICHEL, Agustina. Aborto ¿̇según quién? In: MORÁN FAÚNDES, José Manuel, SGRÓ RUATA, María Candelaria; VAGGIONE, Juan Marco (Eds.). Sexualidades, desigualdades y derechos: reflexiones en torno a los derechos sexuales y reproductivos. Córdoba: Ciencia, Derecho y Sociedad; Ferreyra Editores, 2012. 
SEGHD, Gilda; SINGH, Susheela; SHAH, Iqbal H.; AHMAN, Elisabeth; HENSHAW, Stanley K.; BANKOLE, Akinrinola. Induced abortion: incidence and trends worldwide from 1995 to 2008. The Lancet, London; New York; Beijing, 19 ene. 2012. London; New York; Beijing. <http://www.guttmacher.org/pubs/journals/Sedgh-Lancet-2012-01.pdf > .

SESTA, Michele. Lo privado y lo público en los proyectos de ley en materia de familia. In: CARLUCCI, Aída Kemelmajer (Coord.). El derecho de familia y los nuevos paradigmas. Buenos Aires: Rubinzal-Culzoni Editores, 1999. t. 1.

SMULOVITZ, Catalina. La política por otros medios. Judicialización y movilización legal en la Argentina. Desarrollo Económico, Buenos Aires, v. 48, n. 190/191, p. 287-305. 2008.

UNSAFE Abortion: global and regional estimates of the incidence of unsafe abortion and associated mortality in 2008. 6th ed. Ginebra: Organización Mundial de la Salud, 2011.

Maximiliano Nicolás Campana - Doctor en Derechos y Ciencias Sociales, Universidad Nacional de Córdoba. Miembro del Centro de Investigaciones Jurídicas y Sociales de la Universidad Nacional de Córdoba. Abogado. Córdoba, Argentina. E-mail: maxicampana@gmail.com. 